\section{A novel site of insertion of IS6110 in the moaB3 gene of a clinical isolate of Mycobacterium tuberculosis}

\author{
Suma Sarojini,,,2 G K Madhavilatha, ${ }^{1}$ \\ Smitha Soman, ${ }^{1}$ R Ajay Kumar, ${ }^{1}$ \\ Sathish Mundayoor ${ }^{1}$ \\ 1 Mycobacterial Research Group, \\ Department of Molecular Microbiology, \\ Rajiv Gandhi Centre for Biotechnology, \\ Trivandrum; ' Department of \\ Biotechnology, Christ University, \\ Bangalore, India
}

\section{Abstract}

In Mycobacterium tuberculosis, genomic variation is generated mainly by insertions and deletions rather than by point mutations. RvD5 is one such deletion in M. tuberculosis H37Rv. Previous studies from our laboratory have shown the presence of moaA3 gene in the RvD5 region in a large number of clinical isolates, that is absent in M. tuberculosis $\mathrm{H} 37 \mathrm{Rv}$ and H37Ra. The present study was aimed at investigating the RvD5 locus of the clinical isolates by a detailed PCR analysis. Here we report a new point of insertion of the mobile genetic element, IS6110 in the genome of one clinical isolate of $M$. tuberculosis. The insertion has disrupted the moaB3 gene, one of the ORFs in the RvD5 region, which is involved in the molybdopterin biosynthetic pathway. This insertion of IS6110 in the moaB3 of the clinical isolate is different when compared to the insertion in the moaB3 gene of $M$. tuberculosis $\mathrm{H} 37 \mathrm{Rv}$ where $4 \mathrm{~kb}$ RvD5 region has been lost by homologous recombination and only a truncated form of the gene is present. This finding is of relevance since IS6110 is a major element determining the genome plasticity of $M$. tuberculosis and its numerical and positional polymorphism has always been of special interest.

\section{Introduction}

It is well known that bacterial strains within a single species exhibit variations in their genetic profile. Deletions and rearrangements have been found in different members of the M. tuberculosis complex and have been correlated with attenuation and loss of virulence. ${ }^{1-3}$ Regions of Difference (RD1-RD16) that range in size between 2 and $13 \mathrm{~kb}$ were identified in the members of the $M$. tuberculosis complex by various comparative genome analyses.,5
Six regions i.e., H37Rv-related deletions (RvD1 to RvD5 and M. tuberculosis specific deletion 1- TbD1), are absent in the M. tuberculosis $\mathrm{H} 37 \mathrm{Rv}$ genome relative to other members of the $M$. tuberculosis complex. A major source of genetic polymorphism in $M$. tuberculosis is the insertion and deletion of these RDs.4,6 Most RDs or DNA large sequence polymorphisms (LSP) are considered unique event polymorphisms and have been used to define the six major lineages and several sublineages of M. tuberculosis. ${ }^{7}$

IS6110 is found to mediate a number of genomic rearrangements in $\mathrm{RD}$ and $\mathrm{RvD}$ regions. Examples include deletion of the $7 \mathrm{~kb}$ locus RvD2 in M. tuberculosis $\mathrm{H} 37 \mathrm{Rv}$, which is present in the closely related avirulent derivative $\mathrm{H} 37 \mathrm{Ra},{ }^{6}$ loss of RD2 region encoding the protein antigen MPB64 from some strains of $M$. bovis $\mathrm{BCG}^{2}$ etc. The RvD2 region undergoes great variability in clinical isolates of $M$. tuberculosis and seems to represent a hot-spot for IS6110 transposition events. ${ }^{8}$ Our laboratory has been interested in studying the regions that are deleted in the laboratory strain $M$. tuberculosis $\mathrm{H} 37 \mathrm{Rv}$, but are present in clinical isolates of M. tuberculosis. RvD5 region which comprises several moa genes is one such deletion in the $\mathrm{H} 37 \mathrm{Rv}$ strain. Primers designed to amplify an internal region of RvD5 were expected to give an amplicon of $1254 \mathrm{bp}$. Most of the clinical isolates screened using these primers gave the expected amplicon, except RGTB39. PCR amplification of this isolate gave an amplicon of $2.6 \mathrm{~kb}$. Efforts to characterize this fragment led to the finding of an IS6110 insertion disrupting the moaB3 gene in this region in the clinical isolate RGTB39.

\section{Materials and Methods}

\section{M. tuberculosis strains and DNA isolation}

A total of 105 clinical isolates of $M$. tuberculosis from different parts of Kerala in South India were used for the study. The standard strains included in the study are M. tuberculosis $\mathrm{H} 37 \mathrm{Rv}$, M. tuberculosis $\mathrm{H} 37 \mathrm{Ra}$, M. bovis and $M$. bovis BCG (Pasteur). The clinical isolates are the processed sputum samples of tuberculosis patients grown on LJ medium. These were later subcultured in Middlebrook 7H9 Broth (Difco Laboratories) supplemented with OADC enrichment (Difco Laboratories) and $0.5 \%$ glycerol (USB Corporation) at $37^{\circ} \mathrm{C}$ for four weeks. Biochemical characterization and DNA isolation were carried out as described earlier. $^{1}$

\section{PCR amplification of RvD5 region \\ Primer pairs used in this study are moaFP1}

Correspondence: Dr. Sathish Mundayoor, Scientist G and Dean, Rajiv Gandhi Centre for Biotechnology, Thycaud (P0), Trivandrum 695014, India.

Tel. +91.471.2529512 - Fax. +91.471.2348096.

E-mail: smundayoor@rgcb.res.in

Key words: IS6110, moaB3, Mycobacterium tuberculosis.

Acknowledgements: Suma Sarojini, GK Madhavilatha and Smitha Soman acknowledge the Council for Scientific and Industrial Research (CSIR), Govt. of India for Senior Research Fellowship. We thank Laiza K Paul for excellent technical assistance and Manoj P for assistance in sequencing. No funding was received for this work.

Contributions: SS, experimental work, data analysis and manuscript writing; GKM, IS6110 RFLP performance, manuscript writing and proofing; SSN, experimental work; RAK, critically evaluated the manuscript; SM, study design and supervision, manuscript writing.

Conflict of interest: the authors report no conflicts of interest.

Received for publication: 6 October 2011.

Revision received: 15 July 2011.

Accepted for publication: 15 July 2011.

This work is licensed under a Creative Commons Attribution NonCommercial 3.0 License (CC BYNC 3.0).

@CCopyright S. Sarojini et al., 2011

Licensee PAGEPress, Italy

Microbiology Research 2011; 2:e7

doi:10.4081/mr.2011.e7

5'-CCCATCGTGGTCGTTCACC-3' and moaRP1 5'-CGATGGCAGCGGTTTACAG-3' for the flanking sequences, moaFP2 5'-TGTCATAGGAGCTTCCGACC-3' and moaRP2 5'AACATTCGGCATCATTGCTT-3', and moaFP3 5'AGTTTGGTCATCAGCCGTTC-3' and moaRP3 5'-AACCAAGTCCAGGAGGGAAC-3' for the internal sequences. PCR was performed in a final volume of $30 \mu \mathrm{L}$ using 1.25 units of Taq DNA Polymerase (Promega Corporation) for each reaction. After initial denaturation, amplification was done in a PCR thermal cycler (BioRad) for 35 cycles of $94^{\circ} \mathrm{C} / 1 \mathrm{~min}$, $64^{\circ} \mathrm{C} / 1 \mathrm{~min}, 72^{\circ} \mathrm{C} / 1 \mathrm{~min}$ followed by a final extension of $72^{\circ} \mathrm{C} / 7 \mathrm{~min}$.

\section{Cloning and sequencing}

pGEMT Easy vector (Promega) was used to clone the $2.6 \mathrm{~kb}$ PCR product. Using the six moa PCR primers, template DNA was sequenced using the fluorescent big dye terminator cycle sequencing ready reaction kit (PE Biosystems) in an automated sequencer (ABI Prism 310). The sequencing reaction 
mixtures, each containing $300 \mathrm{ng}$ of template DNA, 3.2 pmol of primer, $2.0 \mu \mathrm{L}$ of Prism Ready Mix and $1.13 \mu \mathrm{L}$ of $25 \mathrm{mM} \mathrm{MgCl}$, were subjected to 25 cycles of denaturation (at $96^{\circ} \mathrm{C}$ for $30 \mathrm{~s}$ ), annealing (at $55^{\circ} \mathrm{C}$ for $15 \mathrm{~s}$ ), and extension (at $60^{\circ} \mathrm{C}$ for $4 \mathrm{~min}$ ). Six different sequencing reactions were carried out i.e., the forward sequences with the three forward primers - moaFP1, moaFP2 and moaFP3 and the reverse sequences with the three reverse primers - moaRP1, moaRP2 and moaRP3.

\section{Aligning sequences and homology search}

DNA sequences identified in this study were compared to sequences in the GenBank and EMBL databases. They were also subjected to BLAST analysis in the M. tuberculosis DNA sequence database (http://genolist.pasteur.fr/ TubercuList) and the M. bovis database (http://genolist.pasteur.fr/BoviList). For DNA sequence alignment, the program BL2SEQ in the SDSC Biology Workbench was utilized.

\section{IS6110 Fingerprinting}

Genomic DNA of the standard strain $M$. tuberculosis $\mathrm{H} 37 \mathrm{Rv}$ and three local isolates was digested with Pvu II (New England Biolabs), separated on $0.8 \%$ agarose gel and blotted onto a nylon membrane (Hybond). After prehybridization at $65^{\circ} \mathrm{C}$ for 3 hours, a 245bp PCR product of IS6110 from M. tuberculosis $\mathrm{H} 37 \mathrm{Rv}$ labelled with $\alpha-{ }^{32} \mathrm{P}$ dCTP probe was denatured, quick chilled and allowed to hybridize the membrane. After overnight hybridization at $65^{\circ} \mathrm{C}$, the blot was washed with increasing stringency of SSC-SDS and exposed to an activated Phosphor screen (Kodak). The screen was then scanned using Personal Molecular Imager FX (BioRad) and the picture was visualized using the software Quantity One (BioRad) to obtain the IS6110 RFLP pattern.

\section{Results}

\section{PCR}

To investigate the RvD5 region and to find out the presence of moaA3 and its adjacent regions in the clinical isolates of $M$. tuberculosis from Kerala, primers were designed to amplify the flanking sequences. One such primer pair (moaFP1 moaRP1) was expected to amplify a region encompassing the whole of moaB3 gene and a part (768bp) of moaA3 gene. The expected size of the PCR product was $1254 \mathrm{bp}$. In our laboratory we have a repository of clinical isolates of $M$. tuberculosis collected from tuberculosis patients in
Kerala. PCR screening of these isolates using the primers moaFP1 and moaRP1 gave the expected product of $1254 \mathrm{bp}$ in all isolates tested. But one of the isolate RGTB39 generated a PCR product of size $2.6 \mathrm{~kb}$ (Figure 1).

\section{Cloning and sequencing of $2.6 \mathrm{~kb}$ PCR product}

The $2.6 \mathrm{~kb}$ band was gel eluted and cloned in pGEMT Easy vector. The ends of the insert were sequenced using moaFP1 and moaRP1 primers. The forward primer gave a sequence which had homology only with 211bp of moaB3. The latter part of the sequence shared homology with a portion of IS6110 transposases seen in the genomes of both $M$. tuberculosis $\mathrm{H} 37 \mathrm{Rv}$ and in M. tuberculosis $\mathrm{CDC1551.} \mathrm{As}$ expected, the sequence obtained using reverse primer had homology with moaA3. To

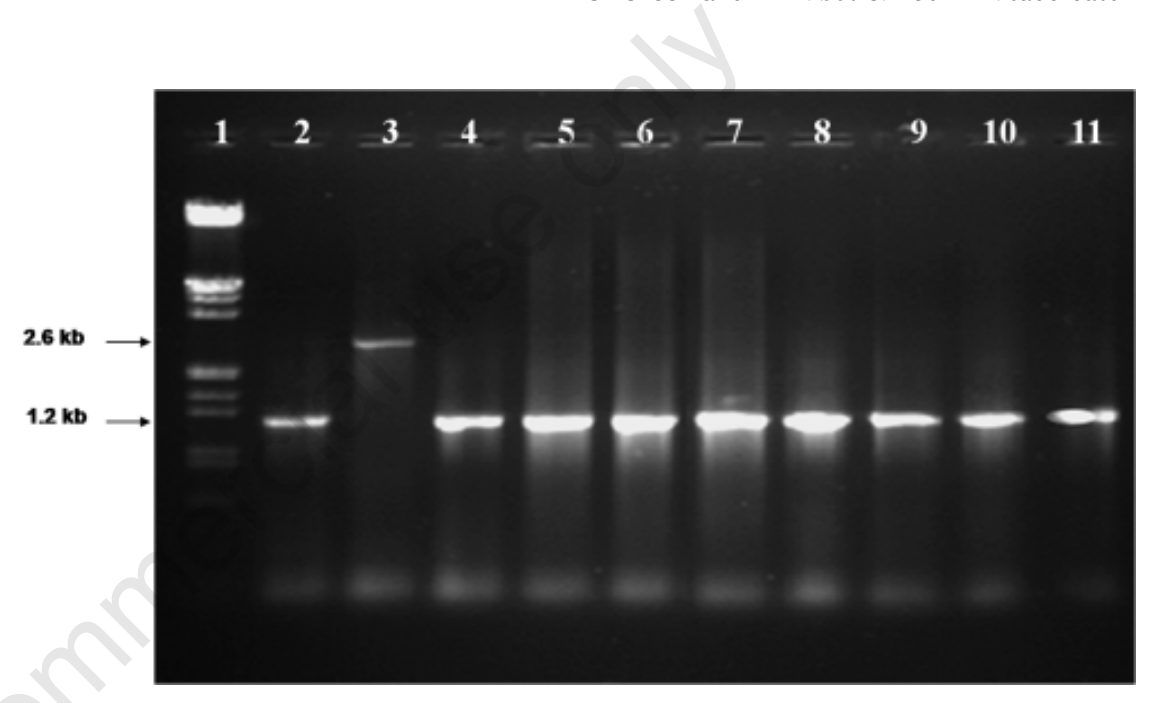

Figure 1. PCR of 10 representative clinical isolates of $M$. tuberculosis from Kerala using primers moaFP1 and moaRP1. Lane 1: Marker DNA (DNA double digest (Hind III / EcoR I); Lanes 2-11: clinical isolates RGTB29, 39, 70, 86, 110, 123. 142, 144, 154 and 177 respectively. RGTB39 shows a $2.6 \mathrm{~kb}$ band and the rest show the $1254 \mathrm{bp}$ band.

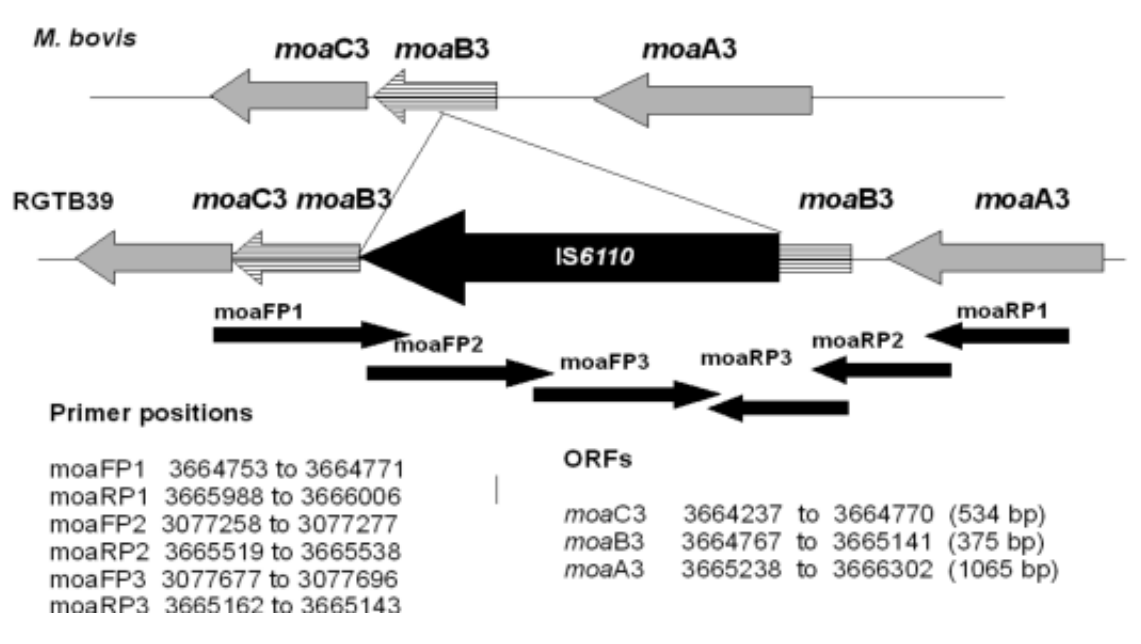

Figure 2. Schematic representation of the genome position of IS6110 insertion in the moa 33 gene of RGTB39. The different sequences obtained to get the final assembly of the $2.6 \mathrm{~kb}$ PCR product are shown as striped arrows. The genome coordinates (M. bovis) of the primer positions and the genes are also shown. 
sis $\mathrm{H} 37 \mathrm{Rv}$ moaB3 is truncated, with only the last $137 \mathrm{bp}$ of the gene being present. The first 238 bp of the moaB3 gene along with the other ORFs comprising the RvD5 region is deleted. Interestingly in RGTB39, none of these scenarios are observed. The IS6110 insertion is after 164 nucleotides and the rest of moaB3 gene is present downstream of IS6110 sequence. Also, the insertion of IS6110 in moaB3 is in the opposite orientation when compared to that in M. tuberculosis H37Rv.

\section{IS6110 RFLP}

IS6110 RFLP of RGTB39, RGTB123 and H37Rv were carried out to find out the number of copies of IS6110 in RGTB39. H37Rv served as the positive control (multicopy IS6110) where as RGTB123 served as the negative control which had no copy of IS6110. We have previously reported a large number of single and no copy strains of $M$. tuberculosis from our region ${ }^{9}$ but RGTB39 turned out to be a strain with more than ten copies of IS6110 (Data not shown).

\section{GenBank Submission}

The 2612bp sequence amplified from RGTB39 using primers moaFP1 and moaRP1 was completely sequenced, analysed and submitted to Genbank (GenBank:GU994139).

\section{Discussion}

Earlier experiments conducted in our laboratory to screen the different RDs and RvDs showed the absence of moaA3, a gene in the RvD5 region, in some of the clinical isolates from Kerala. ${ }^{1}$ Screening larger number of isolates using different primers from RvD5 region showed an IS6110 insertion in the RvD5 region of one clinical isolate, RGTB39. Though initially it seemed to be similar to $M$. tuberculosis $\mathrm{H} 37 \mathrm{Rv}$, it later turned out to be different for three reasons - a) Firstly, the orientation of IS6110 is reverse in RGTB39 when compared to that in M. tuberculosis H37Rv. b) In M. tuberculosis $\mathrm{H} 37 \mathrm{Rv}$, the moaB3 gene is truncated (Rv3324A) with only the last 137 nucleotides being present. The rest of the gene along with three other ORFs is lost in the RvD5 deletion. However in RGTB39 the whole of the moaB3 gene is present flanking the IS6110 insertion. c) The point of insertion of IS 6110 in both the strains is different, since in M. tuberculosis $\mathrm{H} 37 \mathrm{Rv}$ only the last 137 nucleotides remain, whereas in RGTB39 the last 211 nucleotides of the gene are present (Figure 3). Thus, this locus can be considered as a new site of insertion of IS6110. It is interesting to note here that the IS6110 insertion in RGTB39 seems to be an independent event.

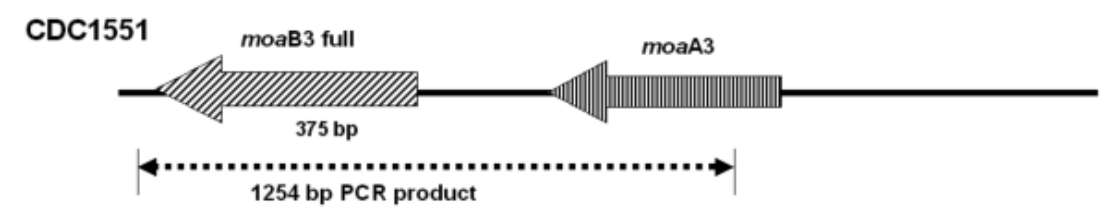

RGTB39

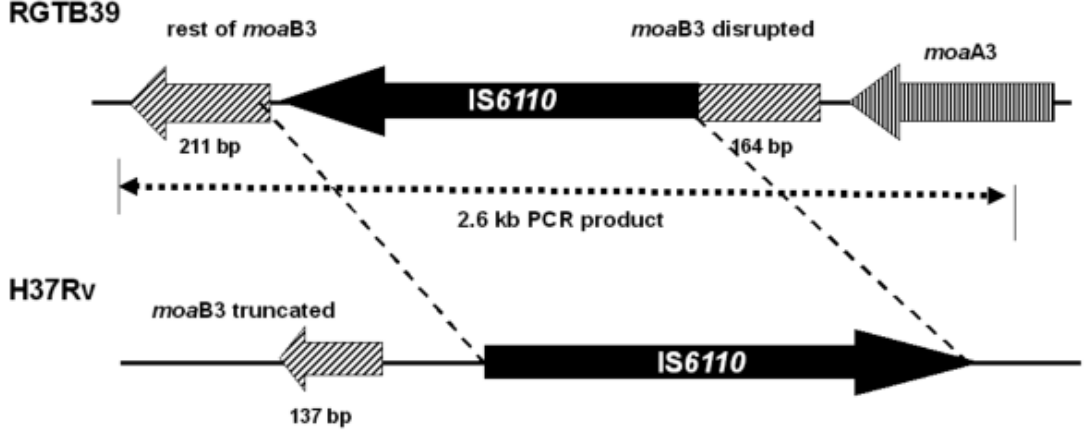

Figure 3. Schematic representation of the comparison of $\mathrm{RvD} 5$ and the flanking regions in $\mathrm{H} 37 \mathrm{Rv}, \mathrm{CDC} 1551$ and RGTB39. In CDC1551 the moaB3 gene is intact. In RGTB39 it is disrupted after $211 \mathrm{bp}$. In H37Rv, the gene is truncated with only a $137 \mathrm{bp}$ fragment in the genome. The region spanning the expected PCR product of $1254 \mathrm{bp}$ in CDC1551 and the region spanning the $2.6 \mathrm{~kb}$ PCR product in RGTB39 are shown in dotted lines.

In clinical isolates, IS6110 preferentially inserts into certain regions of the genome of M. tuberculosis, such as the ipl or the IS preferential locus. Six alternative ipl sequence locations have been reported for the integration of IS6110.10 In addition, SiteMapping, a DNA microarray-based methodology has been used to identify eight previously unknown regions for insertion of IS6110.11 Polymor phisms found in plcD gene in isolates of virulent $M$. bovis were attributed to IS 6110 insertions. ${ }^{12}$ A recent study has described a hotspot for the insertion of IS6110 in the area of the region of difference (RD) $724 .^{13}$

IS6110 transposition events may disrupt open reading frames ${ }^{14}$ or regulatory domains. In addition, IS-mediated deletion events have been suggested to be an important mechanism driving mycobacterial genome variation. ${ }^{6}$ Homologous recombination between directly repeated IS6110 elements has been proposed as a likely mechanism for genomic deletions in clinical isolates. ${ }^{15}$ The integration of an insertion element can result in either up or down regulation of genes in which it resides. One such case where IS6110 acts as a promoter in $M$. tuberculosis infected macrophage has been reported. ${ }^{16}$ It is equally possible that IS6110 insertion may disrupt existing promoter regions leading to down regulation of gene expression. An IS6110 insertion site could possibly be responsible for a strain's increased capacity for transmission and replication. More than $60 \%$ of characterized IS 6110 insertions have been found to disrupt coding regions. But multiple copies of a gene could compensate for natural knockouts created by IS6110 insertion as in the case of PPE (ProPro-Glu) genes of M. tuberculosis. ${ }^{14}$ The nature of any alteration will depend upon the function of the products of the genes involved. Gene knock out studies are needed for the confirmation of the role of such genes on the intracellular survival of the pathogen.

In the present study, the IS6110 insertion was found in moaB3, a gene belonging to the multi gene family of the molybdopterin biosynthetic pathway. Molybdopterin is a cofactor required for nitrate reductase and many other enzymes involved in anaerobic metabolism. Genes involved in the molybdopterin cofactor biosynthesis pathway are present in almost all organisms. A study using a promoter trap vector has identified two of the $M$. tuberculosis genes in this pathway - moaX and moeB1 - as upregulated in the bacteria present in mouse lungs upon infection. ${ }^{17}$ Therefore, moa genes may be important in the intracellular survival of the pathogen. Since the new insertion disrupting moaB3 was found in a clinical isolate obtained from a patient with pulmonary tuberculosis, it could be argued that moaB3 gene may not be essential for pathogenesis, but may play a role in the variation of virulence of clinical isolates. It could also be that other members of the multigene family compensate for the loss of function. Since M. tuberculosis dedicates 21 genes to the biosynthesis of the molybdopterin cofactor $^{18}$ this may be important in the life of the pathogen, but other genes may be expected to complement the loss of activity of moaB3. 


\section{Conclusions}

Genome variation is the main underlying reason for phenotypic differences observed between organisms of the same species. Genome comparison by multiple sequence alignment of six known genomes of mycobacterial strains (CDC1551, F1, C, Haarlem, $\mathrm{H} 37 \mathrm{Rv}$ and $\mathrm{H} 37 \mathrm{Ra}$ ) revealed that most of the variation results from deletion and transposition events preferentially associated with insertion sequences and genes of the PE/PPE (Pro-Glu/Pro-Pro-Glu) family, but not with genes implicated in virulence. The comparison of multiple genomes demonstrates that $M$. tuberculosis genome is currently undergoing an active process of gene decay, analogous to the adaptation process of obligate bacterial symbionts. ${ }^{19}$ So IS6110 mediated genomic rearrangements possibly have a great influence on the generation of genomic variation and ultimately the evolution of M. tuberculosis.

\section{References}

1. Sarojini S, Soman S, Radhakrishnan I, Mundayoor S. Identification of moaA3 gene in patient isolates of Mycobacterium tuberculosis in Kerala, which is absent in M. tuberculosis $\mathrm{H} 37 \mathrm{Rv}$ and H37Ra. BMC Infect Dis 2005;5:81.

2. Mahairas GG, Sabo PJ, Hickey MJ, et al. Molecular analysis of genetic differences between Mycobacterium bovis BCG and virulent M bovis J Bacteriol 1996;178:127482.

3. Tsolaki AG, Hirsh AE, DeRiemer K, et al.
Functional and evolutionary genomics of Mycobacterium tuberculosis: insights from genomic deletions in 100 strains. Proc Natl Acad Sci U S A 2004;101:4865-70.

4. Gordon SV, Brosch R, Billault A, et al. Identification of variable regions in the genomes of tubercle bacilli using bacterial artificial chromosome arrays. Mol Microbiol 1999;32:643-55.

5. Behr MA, Wilson MA, Gill WP, et al. Comparative genomics of BCG vaccines by whole-genome DNA microarray. Science 1999;284:1520-3.

6. Brosch R, Philipp WJ, Stavropoulos E, Colston MJ, Cole ST, Gordon SV. Genomic analysis reveals variation between Mycobacterium tuberculosis $\mathrm{H} 37 \mathrm{Rv}$ and the attenuated M. tuberculosis H37Ra strain. Infect Immun 1999;67:5768-74.

7. Gagneux S, DeRiemer K, Van T, KatoMaeda M, et al. Variable host-pathogen compatibility in Mycobacterium tuberculosis. Proc Natl Acad Sci USA 2006;103:286973.

8. Ho TB, Robertson BD, Taylor GM, et al. Comparison of Mycobacterium tuberculosis genomes reveals frequent deletions in a $20 \mathrm{~kb}$ variable region in clinical isolates. Yeast 2000;17:272-82.

9. Radhakrishnan I, K MY, Kumar RA, Mundayoor S. Implications of low frequency of IS6110 in fingerprinting field isolates of Mycobacterium tuberculosis from Kerala, India. J Clin Microbiol 2001;39: 1683.

10. Fang Z, Forbes KJ. A Mycobacterium tuberculosis IS6110 preferential locus (ipl) for insertion into the genome. J Clin Microbiol 1997;35:479-81.

11. Kivi M, Liu X, Raychaudhuri S, Altman RB,
Small PM. Determining the genomic locations of repetitive DNA sequences with a whole-genome microarray: IS6110 in Mycobacterium tuberculosis. J Clin Microbiol 2002;40:2192-8.

12. Viana-Niero C, Rodriguez CA, Bigi F, et al. Identification of an IS6110 insertion site in plcD, the unique phospholipase $\mathrm{C}$ gene of Mycobacterium bovis. J Med Microbiol 2006;55:451-7.

13. Kim EY, Nahid P, Hopewell PC, Kato-Maeda M. Novel hot spot of IS6110 insertion in Mycobacterium tuberculosis. J Clin Microbiol 2010;48:1422-4.

14. Sampson SL, Warren RM, Richardson M, et al. Disruption of coding regions by IS6110 insertion in Mycobacterium tuberculosis. Tuber Lung Dis 1999;79:349-59.

15. Fang Z, Doig C, Kenna DT, et al. IS6110mediated deletions of wild-type chromosomes of Mycobacterium tuberculosis. J Bacteriol 1999;181:1014-20.

16. Safi H, Barnes PF, Lakey DL, Shams H, Samten B, Vankayalapati R, et al. IS6110 functions as a mobile, monocyte-activated promoter in Mycobacterium tuberculosis. Mol Microbiol 2004;52:999-1012.

17. Dubnau E, Smith I. Mycobacterium tuberculosis gene expression in macrophages. Microbes Infect 2003;5:629-37.

18. Cole ST, Brosch R, Parkhill J, et al. Deciphering the biology of Mycobacterium tuberculosis from the complete genome sequence. Nature 1998;393:537-44.

19. Cubillos-Ruiz A, Morales J, Zambrano MM. Analysis of the genetic variation in Mycobacterium tuberculosis strains by multiple genome alignments. BMC Res Notes 2008;1:110. 